EESTI NSV TEADUSTE AKADEEEMIA TOIMETISED, 28. KÖDE

FOOSIKA * MATEMAATIKA. 1979, NR. 3

ИЗВЕСТИЯ АКАДЕМИИ НАУК ЭСТОНСКОП ССР. ТОМ 28

ФИЗИКА * МАТЕМАТИКА. 1979, № 3

A. SIRK, P. KASK, T. KÄNDLER, T. KARU,

удК $53,089.5$

J. PUSKAR, E. LIPPMAA

\title{
CLIP-CORRELATOR FOR FLUORESCENCE CORRELATION EXPERIMENTS
}

The main sources of data distortion in photon correlation experiments are considered: the detecting system dead time, afterpulses of the photomultiplier tube, instabilities of the exciting laser light intensity and non-uniformity of the correlator multichannel memory counters. A simple clip-correlator designed to minimize the abovementioned sources of error is described.

\section{Introduction}

After introduction in 1969, the digital clip-correlator $\left[{ }^{1}\right]$ has allowed to simplify the processor electronics and decrease the minimum channel width of the «non-stop» real time correlator by some orders of magnitude. During the last ten years, clip-correlators have been used extensively, basically in light scattering experiments for measuring the width of narrow spectral lines (photon correlation spectroscopy) $\left.{ }^{2}\right]$. In addition to the commercial clip-correlators, such as the Malvern K7023 and K7025, some self-built clip-correlators have been described $\left[{ }^{3,4}\right]$. Practically all of these have been designed for light scattering experiments.

However, there exists another kind of experiments requiring measurement of the correlation function of light intensity - the fluorescence correlation (FC) spectroscopy [ $\left.{ }^{5}\right]$. In an FC experiment, the light intensity fluctuations are caused by stochastic fluctuations in the number of observed fluorescent molecules. Such fluctuations can occur due to translational and rotational diffusion or chemical reactions. Since the best achievable time resolution of a real-time full correlator $\left.{ }^{6}\right]$ is insufficient for FC experiments, especially, if rotational diffusion and chemical reaction kinetics are studied, we expect the use of a much faster clip-correlator to be essential in these experiments. It must be noted that a clip-correlator measures the correlation function between the clipped and the undistorted input signals [ $\left.{ }^{1}\right]$. In general, the clipped correlation function differs slightly from the full correlation function. However, in the most important cases, including the FC experiments, the signal form remains undistorted $\left[{ }^{2,7}\right]$ and reduction of the signal-to-noise ratio is almost trifling $\left[{ }^{8}\right]$ (about 1.25 times in the case of an FC experiment $\left[{ }^{7}\right]$ ).

In an FC experiment, the light intensity fluctuations are relatively small as compared to those in a scattering experiment. Typically, the signal (difference between the measured correlation function and the baseline, produced by uncorrelated input signals) is only about $10^{-3}$ 
to $10^{-6}$ in amplitude $\left[{ }^{9}\right]$. To measure the correlation function with sufficient accuracy, the following conditions must be fulfilled. First, a high counting rate (up to $10^{7} \mathrm{cps}\left[{ }^{10}\right]$ ) is required by the signal-tonoise considerations. Second, additive distortions of the correlation function, produced by the light detection system and the processing electronics, must be lower than $10^{-6}$ to $10^{-7}$.

In this paper we shall treat the main sources of distortion in an FC experiment if a digital correlator is used, and describe a simple clipcorrelator, designed and built in an average physical laboratory. Some design alternatives of the system schematics will be discussed.

\section{Distortion in a correlation experiment}

If the light intensity autocorrelation function is measured using a photon counting system (photomultiplier tube + pulse amplifier and discriminator), there are two serious distortion sources contained in the detecting system itself. First, it is the dead time of the detecting system. The light intensity autocorrelation function is proportional to the conditional probability density for the following photocounts if a photon was detected initially. During the dead time of the detecting system no new photocounts can be registered (relative distortion is equal to -1 , see Fig. 1). This dead time includes contributions from the photomultiplier dynode system, overload properties of the amplifier and its transfer characteristics. Even if we assume immediate recovery of the quantum efficiency of the detecting system (a rectangular dead time, as assumed in Fig. 1), during the following time span the counting probability density deviation from the average value is related to the average light intensity, the important parameter being the photon detection rate per dead time, see Fig. 1 .

Second, every photomultiplier tube has a non-zero probability density for a false count (afterpulse) at some given time after the detection of the first photon $\left.{ }^{11}\right]$. This distortion is less important at higher counting rates, in contrast to the first kind of distortion.

Both distortions can be substantially eliminated if the cross-correiation between photocounts in two detecting systems is measured. Alternatively, to eliminate the detecting system dead time distortion, an additional time delay can be used in the non-clipped input of the correlator, equivalent to not accounting for the content of the first channel (or the first few channels), if the channel width (i. e. the sampling time) is comparable with the detecting system dead time.

Fig. 1. Calculated distortions of photon autocorrelation function produced by finite dead time $(h)$ of the photon detector, the dead time supposed to be of rectangular form. The different curves correspond to different mean counting rates (unit being a count per dead time). In calculations, nearly constant light intensity autocorrelation function was supposed, being the case in FC experiment.

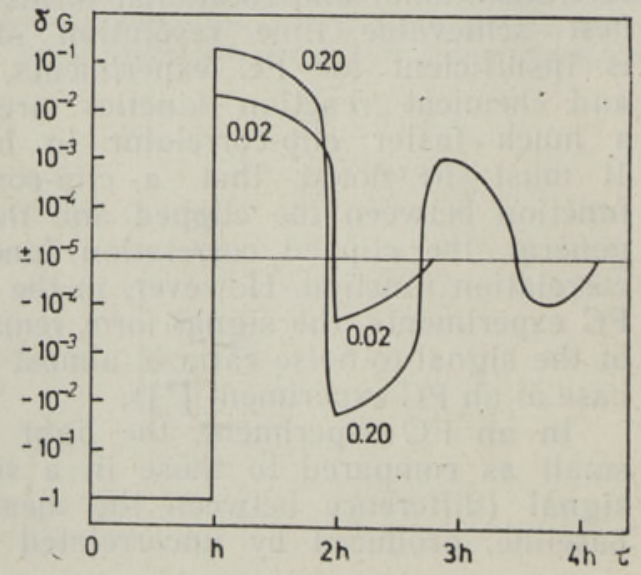


Fig. 2, Block-scheme of the clip-correlator. For simplification, the read-out interface and the control block are not shown.

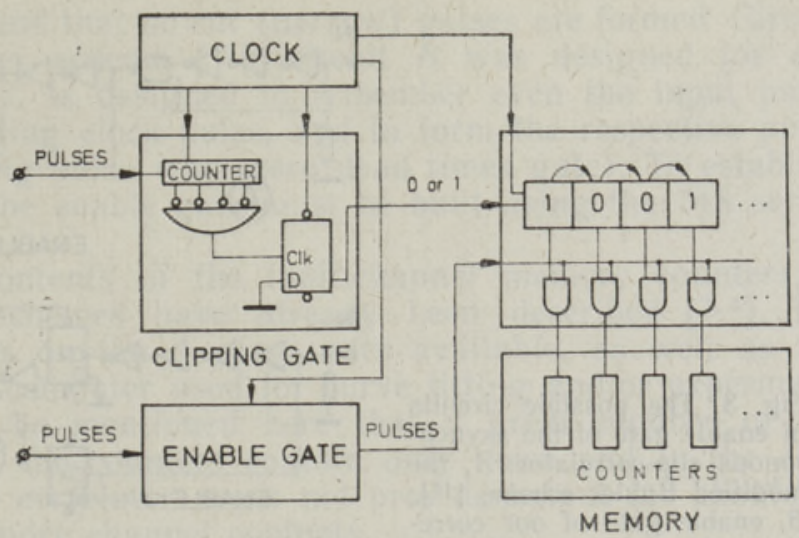

Third, there is a distortion source in both the FC and scattering experiments, not directly related to the correlator itself. This is the noise of the exciting laser intensity. Fortunately, it is not too serious a problem. For example, the laser light instabilities of the order of $10^{-3}$ produce correlation distortions of the order of $10^{-6}$. The laser light can be stabilized with the help of negative feedback circuitry, driving a Pockels or Kerr cell, or the laser power supply. Alternatively, an analog signal, proportional to the laser light intensity, can be produced to drive the sample live time of the correlator (reciprocal dependence on intensity to remove the influence of laser intensity fluctuations) $\left[{ }^{12}\right]$.

Last but not least, we have discovered a serious problem in the design of the correlators - the non-equivalency of the memory channels $\left[{ }^{13}\right]$. One must make sure that all the input pulses, reaching the multiplying AND-gates (see Fig. 2), are counted by all the activated counters (with open AND-gates), but not just by a random number of these. in practice we must guarantee that no pulses arrive at the logic AND-gates during the time interval, when the shift register outputs. are undefined. Also, a minimum pulse width and minimum separation time between any two neighbouring input pulses must be guaranteed. In some cases this problem is solved by synchronizing all the input pulses by the internal clock (pulse derandomization $\left[{ }^{14}\right]$ ). However, if the designer is interested in high counting rates and a good time resolution is less important, then the alternative possibility - gating the non-clipped input pulses - is the better choice.

\section{The correlator}

Among the numerous correlator systems, suitable for FC measurements, we expect our design (see Fig. 2) to be one of the simplest. Basically, it is built using the digital 74 TTL series (Texas Instruments) integrated circuits. To achieve a high counting rate, in some important locations the $74 \mathrm{~S}$ series Shottky elements are used. A 50 to $75 \mathrm{MHz}$ maximum direct counting rate together with a $10 \mathrm{MHz}$ maximum clock frequency of the processor is thus achieved. The $10 \mathrm{MHz}$ clock frequency is chosen to considerably simplify the system design.

The correlator (see Fig. 2) consists of the following main parts: system clock, clipping gate, gating circuitry for the non-clipped input, correlation block with digital delay, the multichannel memory counters, the data output circuit, and the control circuit. 
Fig. 3. The possible circuits of enable gate of the asynchronous clip-correlator. $A$, the modified Buhler scheme $\left[{ }^{16}\right]$; $B$, enable gate of our correlator; $C$, a scheme with onebit memory (the «zero dead time» scheme).
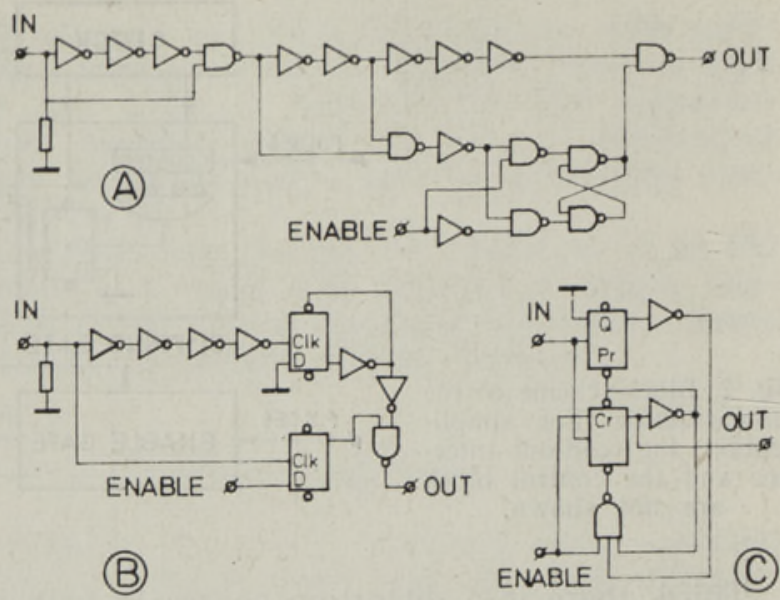

The system clock consists of a $20 \mathrm{MHz}$ quartz oscillator, built around two 74S00 NAND-gates, a frequency divider $(6 \times 74193)$, demultiplexer for selecting the output of the frequency divider and some clock pulse formers with different pulse length, delay and polarity.

The clipping gate consists of a counter $(74 \mathrm{~S} 74$ in the first stages and 74193 thereafter), which can be preset in any given position by the system clock pulse. After a certain number of photon counts, the counter reaches the «zero» state, which is fixed through the logic elements by changing the state of a $74 \mathrm{~S} 74$ trigger (the clipping trigger). For simplified scheme, see the clipping gate in Fig. 2.

Every clock pulse transforms the state of the clipping trigger to the left position of the shift register, the latter serving as the digital delay of the correlation circuit. Multiplying the delayed clipping output of the input pulses by the number of pulses from the non-clipped input is performed on AND-gates of the correlation circuit with following registration in the counters of the memory channels. The fast 74197 counters are used in the first stages of every counter and the slower 74193 counters in the following stages.

The enable gate serves to establish the uniformity of speed of the memory counters (i.e. to eliminate fourth mode of distortion of the previous section). It is the most important circuit in any low distortion correlator for FC experiments. Without special care, distortions of the order of $10^{-3}$ would be common $\left[{ }^{4}\right]$.

An important property of electronic systems, which must be discussed here, is the so-called hang-up of triggers [ $\left.{ }^{15}\right]$. It is a general problem if asynchronous input pulses must be synchronized by the system clock. When the set-up time of the trigger is violated, the bistable may hang in its astable state for an indeterminate time and thus extend the specified time delay. For example, under forced conditions, up to $20 \mathrm{nsec}$ additional delay before output reaches the stable final state, has been observed for a $74 \mathrm{~S} 74$ flip-flop [ $\left.{ }^{15}\right]$. Of course, to minimize the probability of a bistable staying at the point of labile equilibrium, one can build a system with a sharper potential curve maximum, or to shake the potential curve of the system in a defined manner synchronously with the input pulse(s), or use a number of consecutive stages in the synchronizing gate.

In Fig. 3, three different enable gates for the clip-correlator are shown. All these are designed to guarantee that no pulses arrive in the output during the time defined by the gating clock pulses when the shift register 
outputs are undefined, and that no cut (narrow) pulses are formed. Circuit $A$ is a modified Buhler scheme $\left[{ }^{16}\right]$, circuit $B$ was designed for our correlator, and circuit $C$ is designed to remember even the input pulse arriving during the gating clock pulse, and to form the respective pulse after finishing the gating pulse (the «zero dead time» gate). To establish a high counting rate, the enable gate must be built using the $74 \mathrm{~S}$ series IC's.

To read out the contents of the multichannel memory counters, a number of different schemes have already been described $\left[{ }^{3,4}\right]$. The optimal choice depends on the display units available, as well as the input interfaces of the computer used for curve fitting and/or experiment controlling. It should be mentioned here that a great number of the less significant bits of the counters contain only noise and in the case of FC experiments, the correlator needs not provide output for about the first 10 bits of the memory channel contents.

The control circuit design depends upon the needs of the experimentator. As a minimum, it includes the switches to select the sample time and to clear the memory, the switches and a clipmeter (indicating the mean voltage of the clipping gate output) to select the clipping level, the measurement-stand by switch(es), and, probably, a counter to check memory overflow. A correct start and stop of the experiment is best performed by gating the input of the clipping trigger.

The whole correlator, built as described, has a finite (10 to $50 \mathrm{nsec})$ dead time between any two consecutive sample time intervals in both inputs (or in only the clipped one, if the circuit according to Fig. $3 C$ is used). We expect very small dead time to be of negligible importance, at least in the FC experiments.

The main technical data of the FC correlator:

Inputs: minimum pulse width maximum counting rate

Memory: number of channels word length

Time scale: selectable channel width minimum maximum

Clipping level: selectable minimum maximum
$10 \mathrm{nsec}$

$50 \mathrm{MHz}$

32

24 bits

\section{0 nsec}

$0,84 \mathrm{sec}$

0

$2^{19}$ counts

1. J a kem a n, E., P ike, E. R., J. Phys., A2, № 2, $411-412$ (1969).

2. J a keman, E., In: Photon Correlation and Light Beating Spectroscopy, ed. Cummins, H. Z., Pike, E. R., Plenum Press, New York, London, 1974, p. 75-149.

3. Mole, A., Ge is s le r, E., J. Phys. E: Sci. Instrum., 8, № 3, 417-420 (1975).

4. Chen, S. H., Veld kamp, W. B., La i, C. C., Rev. Sci. Instrum., 46, № 10, $1356-1367$ (1975).

5. Els o n, E. L., M a g d e, D., Biopolymers, 13, № 1, 1-27 (1974).

6. H a n, Ch. C., Rev. Sci. Instrum., 49, № 1, 31-38 (1978).

7. Ka sk, P., Kändle r, T, ENSV TA Toim., Füüs. Matem., 27, № 1, $73-78$ (1978).

8. Hughes, A. J., J a kem a n, E., Oliver, C. J., Pike, E. R., J. Phys., A6, № 6, 1327-1331 (1973).

9. M a g d e, D., E l s o n, E. L., W e b b, W. W., Biopolymers, 13, № 1, 29-61 (1974).

10. Ka sk, P., Känd le r, T., S i rk, A., Ka r u, T., Li p p ma a, E., ENSV TA Toim., Füus. Matem., 28, № 3, 221-226 (1979). 
11. Gethner, J. S., F 1 ynn, G. W., Rev. Sci. Instrum., 46, № 5, 586-591 (1975).

12. Kop pel, D. E., Axel rod, D., S chles s inger, J., El son, E. L., Webb, W. W., Biophys. J., 16, № 12, 1315-1329 (1976)

13. Fraser, A., Rev. Sci. Instrum., 42, № 12, 1539-1544 (1971).

14. Oliver, C. J., In: Photon Correlation and Light Beating Spectroscopy, ed. Cummins, H. Z., Pike, E. R., Plenum Press, New York, London, 1974, p. 151 223 .

15. Fi ell a nd, G., O is hi, K., Electron. Des., № 9, 92-94 (1978).

16. Buhle r, O. R., Electron. Des., № 1, $104-106$ (1977).

$$
\begin{gathered}
\text { Academy of Sciences of the Estonian SSR, } \\
\text { Institute of Cybernetics }
\end{gathered}
$$

Received

March 30, 1979

A. SIRK, P. KASK, T. KÄNDLER,

T. KARU, J. PUSKAR, E. LIPPMAA

\section{CLIP-KORRELAATOR \\ FLUORESTSENTSI KORRELATSIOONIEKSPERIMENTIDEKS}

Pōhilised aliikad, mis moonutavad andmeid footonite korrelatsioonieksperimentides, on detekteeriva süsteemi surnud aeg, fotokordisti järelimpulsid, ergastava laserkiirguse ebastabiilsus ja korrelaatori mitmekanalilise mälu loendurite mitteekvivalentsus. On kirjeldatud lihtsat clip-korrelaatorit, mis on konstrueeritud nii, et võimalikult vähendada ülalloetletud vigade allikate mõju.

А. СНРК, П. КАСК, Т. КЯНДЛЕР,

T. КАРУ, Ю. ПУСКАР, Э. ЛИППМАА

\section{КОРРЕЛЯТОР С ПРИВЯЗКОЙ В ОДНОМ КАНАЛЕ ДЛЯ КОРРЕЛЯЦИОННОИ ФЛУОРЕСЦЕНТНОЙ СПЕКТРОСКОПИИ}

Основными источниками искажений в экспериментах по корреляции фотонов являются мертвое время детектирующей системы, послеимпульсы Ф૭่, нестабильность возбуждающего лазерного излучения и неэквювалентность счетчиков многоканальной памяти коррелятора. Описан простой коррелятор с привязкой в одном канале, сконструированный так, чтобы свести до минимума влиянне вышеуказанных источников ошибок. 